\title{
Histopathologic Modification in CNS Neuroectodermal Tumor: A Long Term Follow Up of a Clinical Case
}

\author{
Franceschi $\mathrm{E}^{1 *}$, Bartolotti $\mathrm{M}^{1}$, Brandes $\mathrm{AA}^{1}$, Antonelli $\mathrm{M}^{2}$, Giangaspero $\mathrm{F}^{2,3}$, Tallini $\mathrm{G}^{3}$, Capper $\mathrm{D}^{4}$, \\ Pession $A^{5}$, Scarpelli $\mathbf{M}^{6}$, Vecchioni S $^{7}$, Balestrini ${ }^{8}{ }^{8}$, De Diase $D^{9}$ and Foschini MP ${ }^{10}$ \\ ${ }^{1}$ Department of Medical Oncology, Bellaria-Maggiore Hospitals, Azienda USL - IRCCS, Institute of Neurological Sciences, Bologna, Italy \\ ${ }^{2}$ Department of Radiological, Oncological and Anatomo-Pathological Sciences, Sapienza University, Rome, Italy \\ ${ }^{3}$ IRCCS Neuromed, Pozzilli (Is), Italy
}

${ }^{4}$ Department of Neuropathology, University of Heidelberg, Clinical Cooperation Unit Neuropathology, German Cancer Research Center (DKFZ), Heidelberg, Germany

${ }^{5}$ Department of Medicine (DIMES) Anatomic Pathology-Molecular Diagnostic Unit AUSL of Bologna, University of Bologna School of Medicine, Bologna, Italy.

${ }^{6}$ Section of Pathological Anatomy, Polytechnic University of the Marche Region, School of Medicine, United Hospitals, Ancona, Italy

${ }^{7}$ Department of Neurological Sciences, Section of neurosurgery, United Hospitals, Ancona, Italy

${ }^{8}$ Department of Radiotherapy, Bellaria-Maggiore Hospitals, Azienda USL - IRCCS Institute of Neurological Sciences, Bologna, Italy

${ }^{9}$ Department of Pharmacy and Biotechnology (FaBiT), Molecular Pathology Unit AUSL di Bologna, Bellaria Hospital, University of Bologna, Bologna, Italy

${ }^{10}$ Department of Biomedical and Neuromotor Sciences, Section of Anatomic Pathology, University of Bologna, Bellaria Hospital, Bologna, Italy

*Corresponding author: Enrico Franceschi, Department of Medical Oncology, Bellaria-Maggiore Hospitals, Azienda USL - IRCCS, Institute of Neurological Sciences, Via Altura 3, 40139 Bologna, Italy, Tel: +39 (0)51 6225697; Fax: +39 (0)51 6225057; E-mail: enricofra@yahoo.it

Received: March 12, 2018; Accepted: March 20, 2018; Published: March 22, 2018

Citation: Franceschi E, Bartolotti M, Brandes AA, Antonelli M, Giangaspero F, et al. (2018) Histopathologic Modification in CNS Neuroectodermal Tumor: A Long Term Follow Up of a Clinical Case. Med Case Rep Vol.4 No.2: 64.

\section{Abstract}

Background: Post-therapy differentiation in medulloblastoma (MB) is a rare event that has been described only in pediatric age.

Methods: We describe the long term follow up of a case of medulloblastoma maturation after chemotherapy and radiotherapy in an adult MB patient.

Results and Discussion: A 20-year-old patient was partially resected of a MB with ki-67 of $60 \%$. DNA methylation-derived subgroup showed a molecular $\mathrm{SHH}$ profile. The patient received cranio-spinal tomotherapy (32.4 Gy in 18 fractions) with a boost on posterior fossa (23.4 Gy in 17 fractions, total dose: $55.8 \mathrm{~Gy}$ in 32 fractions). The MRI performed 2 months after revealed a minimal volumetric increase. The patient underwent 2 cycles of Cisplatin - Etoposide chemotherapy. The MRI showed stable disease. The patient underwent second surgery. The specimen showed neuronal cells showing various degrees of maturation from neurocytic to ganglion cells. No embryonal cells were present. Mitoses were absent. The residual lesion showed the post-therapy neuronal maturation of a medulloblastoma. DNA methylation-derived subgrouping confirmed the $\mathrm{SHH}$ profile. No other treatments were delivered after the second resection. To date the patient is still alive and free from progression 54 months after primary surgery.

Conclusion: After a long term follow up, we showed that tumor maturation in an adult patient with medulloblastoma is characterized by a favorable outcome. Physicians should take into account that tumor maturation may occur and that its recognition is essential to define the prognosis and to manage adult patients.

Keywords: Adult medulloblastoma; Differentiation; Radiotherapy; Chemotherapy

\section{Introduction}

Medulloblastoma (MB) is an embryonal tumor of the cerebellum and represents the most common malignant neoplasm of the central nervous system (CNS) in children. MB is rare in adults, (less than $1 \%$ of primitive CNS tumors) with an incidence of $0.6-1$ case per million per year [1]. $M B$ is potentially a curable disease and current treatments allow 5year overall survival (OS) rate of up to $75 \%$. MB comprises a collection of different tumor subgroups: WNT, SHH, Group 3, and Group 4. [2-8]. In adults, the SHH subgroup represents the largest subgroup, accounting for about $60 \%$ of all tumors. The treatment for MB in adults is represented by surgical resection followed by radiotherapy ( $36 \mathrm{~Gy}$ in 20 fractions, followed by a 
boost of $18 \mathrm{~Gy}$ in 10 fractions to the posterior fossa, up to a total of $54 \mathrm{~Gy}$ ), adding chemotherapy for high-risk patients. Post therapeutic neuronal maturation is a rare event that can occur in some pediatric neoplasms, such as neuroblastoma and $M B$ [9-14]. Here we report the long term follow up of an adult $\mathrm{MB}$ that experienced post-therapy neuronal maturation and a favorable outcome.

\section{Case Presentation}

A 20-year-old patient started to experience symptoms with headache and vomiting. The MRI of the brain showed a $4 \times 4$ $\mathrm{cm}$ lesion on the left cerebellar hemisphere. The patient underwent a partial resection of the lesion.

The histopathologic diagnosis was of a nodular/ desmoplastic medulloblastoma (Figures $\mathbf{1 A}$ and 1B). Focal anaplastic changes characterized by enlarged end pleomorphic nuclei with atypical mitoses were also found. Ki-67 reached $60 \%$ in internodular areas and $3 \%$ in other areas.
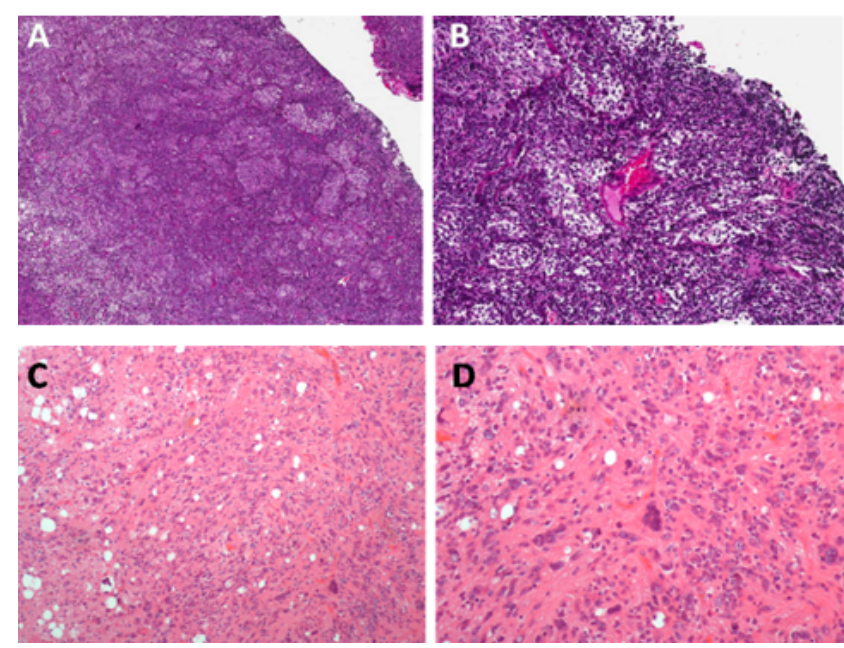

Figure 1 (A and B) Histopathology in the initial medulloblastoma sample, (C and D) Histopathology in medulloblastoma sample and at time of second surgery.

DNA methylation-derived subgroup was performed with Illumina Human Methylation 450 k BeadChip arrays [3]. The lesion showed a molecular SHH profile.

Spinal metastases were excluded by cranio-spinal MRI. Cerebrospinal fluid (CSF) was negative for neoplastic cells. After ovaric tissue preservation the patient received craniospinal tomotherapy (32.4 Gy in 18 fractions) with a boost on posterior fossa (23.4 Gy in 17 fractions, for a total dose of 55.8 Gy in 32 fractions).

The MRI performed 2 months after tomotherapy completion showed a minimal increase of the residual lesion. Thereafter, the patient received 2 cycles of Cisplatin $25 \mathrm{mg} / \mathrm{m} 2$ on days 1 to 4 and Etoposide $40 \mathrm{mg} / \mathrm{m} 2$ on days 1 to 4 every 28 days [4-8]. Disease assessment with brain MRI showed stability of the lesion. Given the refractoriness of the lesion, the patient underwent second surgery. Histopathology showed a neoplasm composed of neuronal cells with various degrees of maturation from neurocytic to ganglion cells, in absence of embryonal cells. No mitoses were found and the Ki-67 was only $2 \%$ (Figures 1C and 1D).

A strong and diffuse positive staining for synaptophisin was observed. The residual lesion was suggestive of a posttreatment neuronal maturation of a medulloblastoma. DNA methylation-derived subgrouping confirmed the molecular $\mathrm{SHH}$ profile. Moreover, chromosomal alterations were similar to the initial lesion. No other treatments were delivered after the second resection. To date the patient is still alive 54 months after primary surgery, without disease relapse.

\section{Discussion}

In this case report we showed that neuronal maturation may occur also in adult MB patients. Tumor maturation is associated with better prognosis in neuroblastoma $[9,10]$ and in pediatric MB [12-14]. Similarly, after a long follow up, our adult patient was alive and free from disease progression. Treatment-induced maturation is a phenomenon that has also been observed in other tumors of embryonal origin, such as embryonal testicular germinal cells tumor (GCT). Indeed, it has been observed that in some cases, following treatment with platin, residual masses have differentiated teratomatous histology [15-20].

However, the mechanism of the maturation is unclear. It is possible that chemotherapy determines the activation/ deactivation of molecular pathways that drives immature cells toward maturation and differentiation [12].

In this case, the diagnosis of tumor maturation, with the absence of mitoses and the reduction of ki67, discouraged the administration of further chemotherapy, given the fact that differentiated cells are more resistant to cytotoxic agents (as has already been seen in testicular cancer maturation) [20].

\section{Conclusion}

In conclusion, we suggest remembering that tumor maturation may happen also in adult patients with neuroectodermic pediatric tumors (i.e. PNET, medulloblastoma) and the recognition of this phenomenon is of importance in managing the patients.

\section{References}

1. Smoll NR, Drummond KJ (2012) The incidence of medulloblastomas and primitive neurectodermal tumours in adults and children. J Clin Neurosci 19: 1541-1544.

2. Taylor MD, Northcott PA, Korshunov A, Remke M, Cho YJ, et al. (2012) Molecular subgroups of medulloblastoma: The current consensus. Acta Neuropathol 123: 465-472.

3. Hovestadt V, Remke M, Kool M, Pietsch T, Northcott PA, et al. (2013) Robust molecular subgrouping and copy-number profiling of medulloblastoma from small amounts of archival tumour material using high-density DNA methylation arrays. Acta Neuropathol 125: 913-916. 
4. Brandes AA, Ermani M, Amista P, Basso U, Vastola F, et al. (2003) The treatment of adults with medulloblastoma: A prospective study. Int J Radiat Oncol Biol Phys 57: 755-761.

5. Brandes AA, Franceschi E (2014) Shedding light on adult medulloblastoma: Current management and opportunities for advances. Am Soc Clin Oncol Educ e82-87.

6. Brandes AA, Franceschi E, Tosoni A, Blatt V, Ermani M (2007) Long-term results of a prospective study on the treatment of medulloblastoma in adults. Cancer 110: 2035-2041.

7. Brandes AA, Franceschi E, Tosoni A, Reni M, Gatta G, et al. (2009) Adult neuroectodermal tumors of posterior fossa (medulloblastoma) and of supratentorial sites (stPNET). Crit Rev Oncol Hematol 71: 165-179.

8. Brandes AA, Paris MK (2004) Review of the prognostic factors in medulloblastoma of children and adults. Crit Rev Oncol Hematol 50: 121-128.

9. Torres LF, Grant N, Harding BN, Scaravilli F (1985) Intracerebral neuroblastoma. Report of a case with neuronal maturation and long survival. Acta Neuropathol 68: 110-114.

10. Kane W, Aronson SM (1967) Gangliogliomatous maturation in cerebellar medulloblastoma. Acta Neuropathol 9: 273-279.

11. De Chadarevian JP, Montes JL, O'Gorman AM, Freeman CR (1987) Maturation of cerebellar neuroblastoma into ganglioneuroma with melanosis. A histologic, immunocytochemical, and ultrastructural study. Cancer 59: 69-76.

12. Cai DX, Mafra M, Schmidt RE, Scheithauer BW, Park TS, et al. (2000) Medulloblastomas with extensive posttherapy neuronal maturation. Report of two cases. J Neurosurg 93: 330-334.
13. Geyer JR, Schofield D, Berger M, Milstein J (1992) Differentiation of a primitive neuroectodermal tumor into a benign ganglioglioma. J Neurooncol 14: 237-241.

14. Valvi S, Ziegler DS (2017) Ganglioglioma arising from desmoplastic medulloblastoma: A case report and review of literature. Pediatrics 139.

15. Gulino A, Arcella A, Giangaspero F (2008) Pathological and molecular heterogeneity of medulloblastoma. Curr Opin Oncol 20: 668-675.

16. Abemayor E, Sidell N (1989) Human neuroblastoma cell lines as models for the in vitro study of neoplastic and neuronal cell differentiation. Environ Health Perspect 80: 3-15.

17. Chang Q, Chen Z, You J, McNutt MA, Zhang T, et al. (2007) Alltrans-retinoic acid induces cell growth arrest in a human medulloblastoma cell line. J Neurooncol 84: 263-267.

18. Bai R, Siu IM, Tyler BM, Staedtke V, Gallia GL, et al. (2010) Evaluation of retinoic acid therapy for OTX2-positive medulloblastomas. Neuro Oncol 12: 655-663.

19. Gumireddy K, Sutton LN, Phillips PC, Reddy CD (2003) All-transretinoic acid-induced apoptosis in human medulloblastoma: activation of caspase-3/poly(ADP-ribose) polymerase 1 pathway. Clin Cancer Res 9: 4052-4059.

20. Abada PB, Howell SB (2014) Cisplatin induces resistance by triggering differentiation of testicular embryonal carcinoma cells. PLoS One 9: e87444. 